\title{
Genetic syndromes associated with overgrowth in childhood
}

\author{
Jung Min Ko, MD, PhD \\ Department of Pediatrics, \\ Seoul National University College of \\ Medicine, Seoul, Korea
}

Overgrowth syndromes comprise a diverse group of conditions with unique clinical, behavioral and molecular genetic features. While considerable overlap in presentation sometimes exists, advances in identification of the precise etiology of specific overgrowth disorders continue to improve clinicians' ability to make an accurate diagnosis. Among them, this paper introduces two classic genetic overgrowth syndromes: Sotos syndrome and Beckwith-Wiedemann syndrome. Historically, the diagnosis was based entirely on clinical findings. However, it is now understood that Sotos syndrome is caused by a variety of molecular genetic alterations resulting in haploinsufficiency of the NSD1 gene at chromosome 5 q35 and that BeckwithWiedemann syndrome is caused by heterogeneous abnormalities in the imprinting of a number of growth regulatory genes within chromosome 11p15 in the majority of cases. Interestingly, the 11 p15 imprinting region is also associated with RussellSilver syndrome which is a typical growth retardation syndrome. Opposite epigenetic alterations in 11 p15 result in opposite clinical features shown in Beckwith-Wiedemann syndrome and Russell-Silver syndrome. Although the exact functions of the causing genes have not yet been completely understood, these overgrowth syndromes can be good models to clarify the complex basis of human growth and help to develop better-directed therapies in the future.

Keywords: Macrosomia, Sotos syndrome, Beckwith-Wiedemann syndrome, genomic imprinting

\section{Introduction}

Somatic growth is dependent on an increase in both cell size and number. Human growth comes from a complex interplay of various factors including genetic backgrounds and environmental influences. However, complex mechanisms involved in the regulation of somatic growth remains to be completely understood particularly in the molecular level of genetic field. Overgrowth refers to a condition characterized by extreme physical size and stature including tall stature or generalized/localized overgrowth of tissues ${ }^{1)}$. The condition originates during infancy, childhood or adolescence while epiphyseal growth plates remain open. Among various conditions showing overgrowth, genetic overgrowth syndrome refers to a nonhormonally mediated overgrowth condition which can accompany increased height and/or head circumference, various degrees of mental retardation, or physical dysmorphisms in children ${ }^{1)}$. It is clearly distinguished from pituitary gigantism which is caused by excess of growth hormone. However, there are overlaps in clinical and molecular features between overgrowth syndromes, thus making a specific diagnosis is often difficult.

This paper reviews clinical characteristics and molecular basis of typical genetic overgrowth syndromes, focusing on Sotos syndrome (OMIM\#117550) and Beckwith-Wiedemann syndrome (OMIM\#130650).

\author{
Address for correspondence: \\ Jung Min Ko, MD, PhD \\ Department of Pediatrics, \\ Seoul National University Children's \\ Hospital, Seoul National University \\ College of Medicine, 101 Daehak-ro, \\ Jongno-gu, Seoul 110-744, Korea \\ Tel: +82-2-2072-3570 \\ Fax: +82-2-743-3455 \\ E-mail:jmko@snu.ac.kr
}

apem
Received: 20 August, 2013
This is an Open Access article distributed under the terms of the Creative Commons Attribution Non-Commercial License (http:// creativecommons.org/licenses/by-nc/3.0) which permits unrestricted non-commercial use, distribution, and reproduction in any medium, provided the original work is properly cited.
ISSN: 2287-1012(Print) ISSN: 2287-1292(Online) 


\section{Sotos syndrome}

\section{Clinical characteristics}

Sotos syndrome (SS, OMIM\#117550), also known as cerebral gigantism, is a prenatal and postnatal overgrowth syndrome characterized by excessive growth resulting in tall stature and macrocephaly, distinctive craniofacial features, and developmental delay. These three cardinal features are each present in over $90 \%$ of cases with $\mathrm{SS}^{2,3}$. Since the first description by Sotos et al. ${ }^{4}$ in 1964, hundreds of cases have been reported to date, and the estimated incidence is $1 / 15,000-1 / 20,000^{1)}$.

The typical overgrowth pattern of SS starts prenatally, resulting in higher mean birth length and weight ${ }^{5}$. Pronounced postnatal growth is obvious in the first 6 years of life, consistently displaying height above the 97 th percentile ${ }^{6}$. However, the final adult height is usually within the upper normal range due to accompanied bone age advancement $t^{6)}$.

A characteristic facial appearance consists of a high and broad forehead, sparse fronto-temporal hair, malar flushing, down-slanted palpebral fissures and a pointed $\operatorname{chin}^{7}$. The head circumference is increased above the 97 th percentile in most SS patients, and it is thought to be the most consistent indicator of SS at any age $e^{5)}$.

The majority of SS patients have some degree of developmental delay/learning disability. Achievement of developmental milestones such as walking and speech is commonly delayed. However, most patients have mild to moderate intellectual impairment, and the severity is very broad, ranging from intelligence quotient below 30 to above $100^{2}$.

Besides, there are other commonly associated features including a history of neonatal jaundice and feeding difficulty, variable types of cardiac and renal anomalies, seizure, scoliosis, strabismus, attention deficit hyperactivity disorder, nonspecific abnormal brain image findings such as ventriculomegaly and corpus callosum hypoplasia. Patients with overgrowth syndromes including SS have higher risks for the development of neoplasias, particularly in their childhood. In SS patients, the frequency of tumor development has been reported to be $2-7 \%^{8,9)}$, and the relative risk of certain malignancies including neural crest tumors, saccrococcygeal teratomas and some hematological malignancies is increased ${ }^{2}$. However, routine screening of tumor development is not a standardized recommendation.

\section{Molecular and genetic basis}

In 2002, the nuclear receptor set domain containing protein 1 gene, $N S D 1$, on chromosome $5 \mathrm{q} 35$ was identified as a causing gene of SS ${ }^{10)}$. SS is caused by haploinsufficiency of NSD1 in 60$90 \%$ of clinically diagnosed SS patients and can be transmitted in an autosomal dominant manner, although more than $95 \%$ of patients gain the disease from de novo mutation ${ }^{11}$.

The NSD1 gene consists of 23 exons and encodes multiple functional domains, including the SU(VAR)3-9, E(Z), tirthorax (SET), SET-associated domains, which mediate histone methyltransferase activity, five plant homeo-domains implicated in chromatin regulation, and two proline-tryptophantryptophan-proline domains that may mediate protein interactions ${ }^{3)} . N S D 1$ is expressed in several tissues including the brain, kidney, skeletal muscle, spleen, and thymus ${ }^{12}$. Although the exact role of the NSD1 protein has not been identified, the presence of two different ligand binding domains suggests that NSD1 enables the regulation of both negative and positive transcription ${ }^{13)}$.

Several reports have demonstrated NSD1 abnormalities in patients with Sotos syndrome. NSD1 abnormalities include microdeletion of $5 \mathrm{q} 35$, encompassing the entire NSDI deletions
Japanese Sotos patients

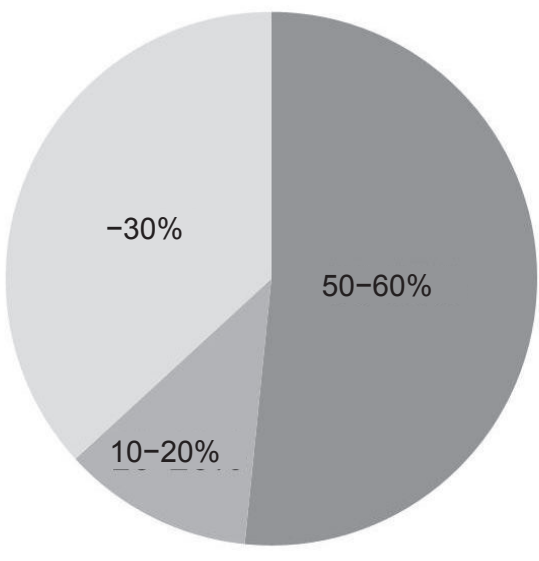

$5 q 35$ microdeletion

NSD1 intragenic mutation

No abnormality
Non-Japanese Sotos patients

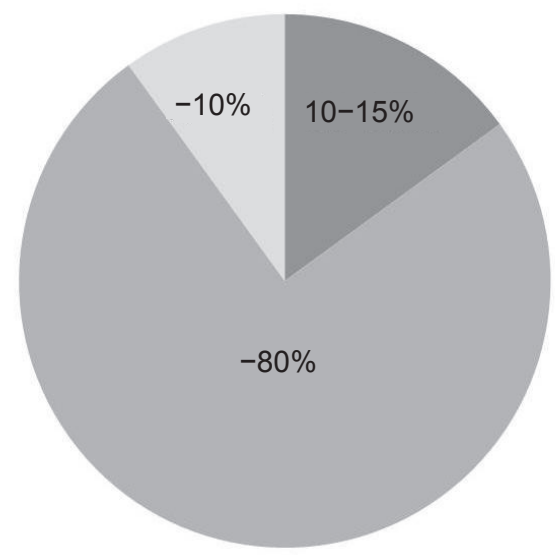

Fig. 1. Chromosome $5 q 35$ microdeletions are more frequently found in Japanese patients with Sotos syndrome, whereas $5 q 35$ microdeletions are uncommon in patients outside of Japan. 
and mutations within the NSD1 gene (intragenic mutations). To date, over 300 different mutations associated with Sotos syndrome have been identified, including gross deletions, small indels, point mutations, and splice site mutations (Human Gene Mutation Database, http://www.hgmd.org/). Notably, $5 q 35$ microdeletions are more frequently (approximately 50\%) found in Japanese patients with Sotos syndrome ${ }^{10)}$, whereas 5 q35 microdeletions are uncommon $(<15 \%)$ in patients in other areas of the world ${ }^{3)}$ (Fig. 1). Recently, the first report of SS in Korea was published ${ }^{14)}$, and this study documented that $53 \%$ of patients had a $5 \mathrm{q} 35$ microdeletion, a result that is very similar to that in Japan. Moreover, NSD1 abnormalities have been delineated in up to $90 \%$ of non-Japanese patients with Sotos syndrome, whereas approximately $30 \%$ of Japanese and Korean patients do not have NSD1 abnormalities ${ }^{14,15}$. 5 q35 microdeletions can be detected by fluorescence in situ hybridization (FISH), multiplex ligation dependent probe amplification (MLPA), or array comparative genomic hybridization methods (array CGH), and NSD1 intragenic mutations can be identified by the direct sequencing method using the patient's genomic DNA.

However, the exact mechanism of such an interracial difference has not been clearly explained to date. Patients with a microdeletion tend to have certain congenital heart and/ or urogenital anomalies, more severe mental retardation, and shorter stature than those with NSDI intragenic mutations ${ }^{16)}$.

\section{Beckwith-Wiedemann syndrome}

\section{Clinical characteristics}

Beckwith-Wiedemann syndrome (BWS, OMIM\#130650) is the most common genetic overgrowth syndrome, with an estimated incidence of $1 / 13,700^{17)}$. However, the actual incidence may be higher than the estimated incidence, considering the broad clinical spectrum of $\mathrm{BWS}^{18)}$.

This syndrome is characterized by prenatal and postnatal overgrowth, polyhydramnios, abdominal wall defects including omphalocele, macroglossia, and visceromegaly of abdominal organs, hemihyperplasia, neonatal hyperinsulinemic hypoglycemia, ear creases and pits, cleft palate, and predisposition to embryonal tumors ${ }^{19)}$. These clinical features are quite variable, and there are no absolute criteria for a clinical diagnosis to date. Although intelligence is usually normal, mild to moderate developmental delay can be found in patients with a history of hypoglycemia and airway problems ${ }^{19)}$.

Because of the well documented association between BWS and increased risk of embryonal tumor development, early diagnosis of BWS is important in the clinical setting. Wilms tumor and hepatoblastoma are most commonly accompanied, and various malignancies including neuroblastoma, rhabdomyosarcoma, adrenocortical carcinoma, and benign tumors have also been reported in BWS patients ${ }^{20,21)}$. The published lifetime incidence of embryonal tumors in BWS patients varies between 4 and $21 \%$ (mean, $7.5 \%$ ), and most of the tumors are present in the first 8 years of life $\mathrm{e}^{19)}$. Therefore, tumor surveillance is particularly targeted at detecting Wilms tumor and hepatoblastoma, and abdominal ultrasonography during infancy and early childhood is highly recommended to all BWS patients $^{22)}$. Tumor surveillance may reduce treatment-related morbidities if tumors can be detected at early stages.

Various protocols for tumor surveillance have been proposed $^{20,23,24)}$. Although there are no established consensus guidelines for tumor surveillance in BWS, most previous studies suggest abdominal ultrasonography every 3-6 months to the age of 8 years and measuring serum alpha fetoprotein every $2-3$ months until 4 years ${ }^{20,23,24)}$.

\section{Molecular and genetic basis}

The heterogeneous molecular basis for BWS has been studied since the mapping of chromosome 11 p15 as the causative locus for BWS by Waziri et al. ${ }^{25)}$ in 1983. However, due to the genetic complexity in BWS, the molecular diagnosis of BWS is still difficult, and the diagnosis of BWS mainly relies on clinical features.

BWS is caused by dysregulation of imprinted growth regulatory genes on chromosome $11 \mathrm{p} 15^{26)}$. In contrast to most autosomal genes, only one allele of either the paternal or maternal allele in each imprinted gene is expressed normally. It is a parental origin-specific manner. Abnormalities in the imprinting process of a number of growth regulatory genes within the two domains on 11p15 lead to dysregulation of prenatal and postnatal growth and cause BWS ${ }^{19)}$.

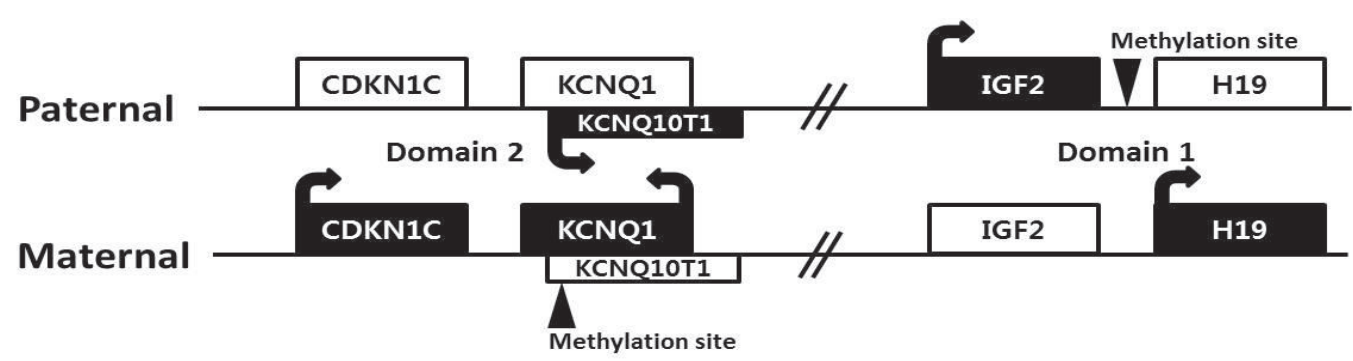

Fig. 2. Chromosome 11 p15 region has two imprinting domains (domain 1 and 2) and four major genes (IGF2, H19, CDKN1C, and KCNQ10T1) associated with genomic imprinting. 
There are four major genes $(I G F 2, H 19, C D K N 1 C$, and $K C N Q 10 T 1)$ associated with genomic imprinting in two imprinting domains (imprinting domain 1 and 2) on $11 \mathrm{p} 15$, and expression of these genes in each domain is cis-regulated by imprinting centers 1 and 2, respectively (Fig. 2). IGF2 and H19 are located in domain 1, and CDKN1C and KCNQ10T1 are located in domain 2. Normally, IGF2 is a paternally expressed gene and encodes insulin-like growth factor 2, which is the major growth factor in utero that promotes prenatal growth. H19 is a maternally expressed gene and encodes an untranslated mRNA, which may function as a tumor suppressor and a growth restrictor ${ }^{27,28)}$. CDKN1C is a maternally expressed gene and encodes a cyclin-dependent kinase inhibitor that negatively regulates cell proliferation ${ }^{29)}$. KCNQ10T1 is a paternally expressed gene and encodes untranslated mRNA, which may act as a negative regulator for $C D K N 1 C$ expression $^{30)}$. Therefore, paternally expressed genes (IGF2 and KCNQ10T1) promote growth particularly in utero, and maternally expressed genes (H19 and CDKN1C) restrict growth, opposing the effect of paternally expressed genes.

In BWS, alterations of these imprinting processes in the genes of domain 1 and 2 can be molecularly identified in approximately $70 \%$ of patients by various kinds of methylation studies ${ }^{19)}$. Approximately $50 \%$ of patients show hypomethylation of domain 2 in the maternal allele, causing expression of maternal KCNQ10T1. Increased expression of IGF2 occurs in approximately $25 \%$ of patients via paternal uniparental disomy (20\%) or hypermethylation of domain 1 in the maternal allele (5\%). The broad clinical spectrum of BWS is likely associated with the heterogeneity of these underlying genetic mechanisms. Furthermore, approximately $5 \%$ of patients have intragenic mutations in CDKN1C of maternal allele. However, more than $20 \%$ of BWS patients still remain with undetermined molecular causes $^{19)}$. Methylation-specific MLPA is currently the most robust method to confirm BWS, and it can detect methylation defects of domain 1 or 2 (55\%), paternal uniparental disomy (20\%), and microdeletion/microduplication involving domain 1 and/or 2 (rare). Cytogenetically detectable abnormalities using conventional karyotyping and FISH analysis are found only less than $1 \%$ in BWS patients. Sequence analysis of the CDKN1C gene can identify intragenic mutations $(5 \%)^{31)}$.

Interestingly, the $11 \mathrm{p} 15$ imprinting region is also associated with Russell-Silver syndrome (RSS, OMIM\#180860), which is a typical growth retardation syndrome ${ }^{32,33)}$. In contrast to BWS, silencing paternally expressed genes (IGF2 and KCNQ10T1) or enhancing maternally expressed genes (H19 and CDKN1C) leads to RSS ${ }^{34)}$. Opposite epigenetic alterations in $11 \mathrm{p} 15$ result in opposite clinical features shown in BWS and RSS. Alterations in the imprinting process on 11 p 15 can be identified in approximately $50 \%$ of RSS patients ${ }^{32,33)}$.

\section{Conclusions}

Human growth ensues from a complex interplay of genetic traits and environmental influences. In particular, basic studies have continued to clarify the molecular basis of growth disorders in the genetic field. Although the exact functions of the causing genes have not yet been completely understood, genetic overgrowth syndromes can be good models to clarify the complex basis of human growth and assist with the development of better-directed therapies in the future.

\section{Conflict of interest}

No potential conflict of interest relevant to this article was reported.

\section{Acknowledgments}

We thank the patients and their families for participating in this study, which was supported by a grant no. NRF2012R1A1A3001588 from the Korean Ministry of Science, ICT and Future Planning, and a grant of the Korean Society of Pediatric Endocrinology.

\section{References}

1. Neylon OM, Werther GA, Sabin MA. Overgrowth syndromes. Curr Opin Pediatr 2012;24:505-11.

2. Tatton-Brown K, Rahman N. Sotos syndrome. Eur J Hum Genet 2007;15:264-71.

3. Tatton-Brown K, Douglas J, Coleman K, Baujat G, Cole TR, Das S, et al. Genotype-phenotype associations in Sotos syndrome: an analysis of 266 individuals with NSD1 aberrations. Am J Hum Genet 2005;77:193-204.

4. Sotos JF, Dodge PR, Muirhead D, Crawford JD, Talbot NB. Cerebral gigantism in childhood: a syndrome of excessively rapid growth and acromegalic features and a nonprogressive neurologic disorder. N Engl J Med 1964;271:109-16.

5. Cole TR, Hughes HE. Sotos syndrome: a study of the diagnostic criteria and natural history. J Med Genet 1994;31:20-32.

6. Agwu JC, Shaw NJ, Kirk J, Chapman S, Ravine D, Cole TR. Growth in Sotos syndrome. Arch Dis Child 1999;80:33942.

7. Allanson JE, Cole TR. Sotos syndrome: evolution of facial phenotype subjective and objective assessment. Am J Med Genet 1996;65:13-20.

8. Wit JM, Beemer FA, Barth PG, Oorthuys JW, Dijkstra PF, Van den Brande JL, et al. Cerebral gigantism (Sotos syndrome). Compiled data of 22 cases. Analysis of clinical features, growth and plasma somatomedin. Eur J Pediatr 1985;144:131-40.

9. Hersh JH, Cole TR, Bloom AS, Bertolone SJ, Hughes HE. Risk of malignancy in Sotos syndrome. J Pediatr 1992;120(4 Pt 1):572-4.

10. Kurotaki N, Imaizumi K, Harada N, Masuno M, Kondoh T, Nagai T, et al. Haploinsufficiency of NSD1 causes Sotos syndrome. Nat Genet 2002;30:365-6. 
11. de Boer L, Kant SG, Karperien M, van Beers L, Tjon J, Vink GR, et al. Genotype-phenotype correlation in patients suspected of having Sotos syndrome. Horm Res 2004;62:197-207.

12. Kurotaki N, Harada N, Yoshiura K, Sugano S, Niikawa N, Matsumoto N. Molecular characterization of NSD1, a human homologue of the mouse Nsd 1 gene. Gene 2001;279:197-204

13. Huang N, vom Baur E, Garnier JM, Lerouge T, Vonesch JL, Lutz Y, et al. Two distinct nuclear receptor interaction domains in NSD1, a novel SET protein that exhibits characteristics of both corepressors and coactivators. EMBO J 1998;17:3398-412.

14. Sohn YB, Lee CG, Ko JM, Yang JA, Yun JN, Jung EJ, et al. Clinical and genetic spectrum of 18 unrelated Korean patients with Sotos syndrome: frequent 5 q 35 microdeletion and identification of four novel NSD1 mutations. J Hum Genet 2013;58:73-7.

15. Niikawa N. Molecular basis of Sotos syndrome. Horm Res 2004;62 Suppl 3:60-5.

16. Nagai T, Matsumoto N, Kurotaki N, Harada N, Niikawa N, Ogata T, et al. Sotos syndrome and haploinsufficiency of NSD 1: clinical features of intragenic mutations and submicroscopic deletions. J Med Genet 2003;40:285-9.

17. Engstrom W, Lindham S, Schofield P.Wiedemann-Beckwith syndrome. Eur J Pediatr 1988;147:450-7.

18. Greer KJ, Kirkpatrick SJ, Weksberg R, Pauli RM. BeckwithWiedemann syndrome in adults: observations from one family and recommendations for care. Am J Med Genet A 2008;146A:1707-12.

19. Choufani S, Shuman C, Weksberg R. Beckwith-Wiedemann syndrome. Am J Med Genet C Semin Med Genet 2010;154C:343-54.

20. Tan TY, Amor DJ. Tumour surveillance in BeckwithWiedemann syndrome and hemihyperplasia: a critical review of the evidence and suggested guidelines for local practice. J Paediatr Child Health 2006;42:486-90.

21. Cohen MM Jr. Beckwith-Wiedemann syndrome: historical, clinicopathological, and etiopathogenetic perspectives. Pediatr Dev Pathol 2005;8:287-304.
22. DeBaun MR, Tucker MA. Risk of cancer during the first four years of life in children from The Beckwith-Wiedemann Syndrome Registry. J Pediatr 1998;132(3 Pt 1):398-400.

23. Clericuzio CL, Martin RA. Diagnostic criteria and tumor screening for individuals with isolated hemihyperplasia. Genet Med 2009;11:220-2.

24. Zarate YA, Mena R, Martin LJ, Steele P, Tinkle BT, Hopkin RJ. Experience with hemihyperplasia and BeckwithWiedemann syndrome surveillance protocol. Am J Med Genet A 2009;149A:1691-7.

25. Waziri M, Patil SR, Hanson JW, Bartley JA. Abnormality of chromosome 11 in patients with features of BeckwithWiedemann syndrome. J Pediatr 1983;102:873-6.

26. Weksberg R, Shuman C, Smith AC. Beckwith-Wiedemann syndrome. Am J Med Genet C Semin Med Genet 2005;137C:12-23.

27. Hao Y, Crenshaw T, Moulton T, Newcomb E, Tycko B. Tumour-suppressor activity of H19 RNA. Nature 1993;365:764-7.

28. Guo L, Choufani S, Ferreira J, Smith A, Chitayat D, Shuman $\mathrm{C}$, et al. Altered gene expression and methylation of the human chromosome 11 imprinted region in small for gestational age (SGA) placentae. Dev Biol 2008;320:79-91.

29. Tsugu A, Sakai K, Dirks PB, Jung S, Weksberg R, Fei YL, et al. Expression of p57(KIP2) potently blocks the growth of human astrocytomas and induces cell senescence. Am J Pathol 2000;157:919-32.

30. Cerrato F, Vernucci M, Pedone PV, Chiariotti L, Sebastio $\mathrm{G}$, Bruni CB, et al. The $5^{\prime}$ end of the KCNQ1OT1 gene is hypomethylated in the Beckwith-Wiedemann syndrome. Hum Genet 2002;111:105-7.

31. Weksberg R, Shuman C, Beckwith JB. BeckwithWiedemann syndrome. Eur J Hum Genet 2010;18:8-14.

32. Eggermann T, Begemann M, Binder G, Spengler S. SilverRussell syndrome: genetic basis and molecular genetic testing. Orphanet J Rare Dis 2010;5:19.

33. Eggermann T. Russell-Silver syndrome. Am J Med Genet C Semin Med Genet 2010;154C:355-64.

34. Eggermann T. Silver-Russell and Beckwith-Wiedemann syndromes: opposite (epi)mutations in 11 p 15 result in opposite clinical pictures. Horm Res 2009;71 Suppl 2:30-5. 